and intracellular level. This inhibition was more pronounced after polyphenol cell pretreatment. In this case cytokine production was completely suppressed both in control and in stimulated cells. Cell pretreatment was extremely effective also in reducing mRNA expression of IL-1 after crystal stimulation while NLRP3 expression was to some extent affected by RES. RES and PD had a slight inhibitory effect on crystal phagocytosis when added along with the stimulus, while pretreated cells did not show any difference in the phagocytosis index. ROS production induced by MSU crystals was more pronounced (4 fold increase) with respect to CPP crystals (2 fold increase). RES was more effective than PD in inhibiting ROS production ( $p<0.05$ crystals vs crystals + RES). The pretreatment showed a more marked decrease of ROS than the simultaneous treatment and the effect reached significance for both PD and RES. Similar inhibitory effects have been obtained when NO production was considered.

Conclusions: Our results demonstrated that RES and PD are effective in inhibiting crystal-induced inflammation affecting IL-1 production and NLRP3 expression and activation. Data obtained after cell pretreatment allow us to hypothesize that these polyphenols act on specific signaling pathway preventing inflammation, and that this action is independent from crystal phagocytosis.

Disclosure of Interest: None declared

DOI: 10.1136/annrheumdis-2017-eular.6830

\section{THU0048 SOLUBLE URIC ACID INCREASED THE EXPRESSION OF ABCG2 AND PDZK1 IN HUMAN INTESTINAL CELL LINES THROUGH THE TLR4/NLRP3/CASPASE-1 AND PI3K/AKT SIGNALING PATHWAYS}

M. Chen, X. Lu, H. Wu. Department of Rheumatology, the Second Affiliated Hospital Zhejiang University School of Medicine, Hangzhou, China

Background: Hyperuricemia is the key pathophysiological basis of gout ${ }^{[1]}$. The most common reason generating hyperuricemia is verified the obstacle of urate excretion by current researches ${ }^{[2]}$. The intestine is known as the most important organ involved the excretion of uric acid besides the kidney ${ }^{[1]}$. ABCG2 has an outsized genetic contribution to extra-renal underexcretion and causes a compensatory increase in urinary urate output ${ }^{[3]}$. PDZK1 is a kind of an important structural protein regulated $A B C G 2$ function, by potential molecular interaction with $A B C G 2^{[4]}$

Objectives: The present study was undertaken to explore the effect and its related mechanisms of soluble uric acid on the urate excretion PDZK1 and ABCG2 in HT-29 and Caco-2 cell lines

Methods: HT-29 and Caco-2 cell lines were used as a well-established model of human intestinal epithelial cells. Cells were pretreated with or without inhibitors and then stimulated with soluble uric acid. siRNA transfection was used to assess the interaction between ABCG2 and PDZK1. qRT-PCR and western blotting were used to measure mRNA and protein levels, respectively. Subcellular fractionation methods and immunofluorescence were used to examine the proteins in different subcellular compartments. Flow cytometry experiments examined the function of ABCG2.

Results: Soluble uric acid significantly up regulated the expression of PDZK1 and ABCG2 in human intestinal cell lines. It also activated TLR4/NLRP3/caspase-1 inflammasome and PI3K/AKT signalling pathway through the phosphorylation of the ser473 pAkt. The increased expression of PDZK1 and ABCG2 was suppressed by a block of TLR4 (TAK-242) and caspase-1 inhibitor (acetyl-YVADchloromethylketone) and partly reduced by wortmanning, a specific inhibitior of PI3K. Additionally, lipopolysaccharide (LPS), the potent inducer of inflammatory responses mediated through TLR4, activated TLR4/NLRP3/caspase-1 inflammasome and up regulated the expression of $A B C G 2$ and PDZK1. Besides, the stimulation of soluble uric acid facilitated the translocation of ABCG2 from intracellular compartment to plasma membrane and increased the transport activity. Furthermore, PDZK1 knockdown via siRNA significantly inhibited the expression and transport activity of $A B C G 2$ regardless of the activation by soluble uric acid. Conclusions: The PI3K/AKT and TLR4/NLRP3/caspase-1 signaling pathways modulate ABCG2 and PDZK1 expression stimulated by soluble uric acid in human intestinal cells. PDZK1 plays a pivotal role in the regulation of ABCG2.

References:

[1] Dalbeth N, Merriman TR, Stamp LK. Gout. Lancet. 2016;388(10055):2039-52.

[2] Perez-Ruiz F, Calabozo M, Erauskin GG, Ruibal A, Herrero-Beites AM. Renal underexcretion of uric acid is present in patients with apparent high urinary uric acid output. Arthritis and rheumatism. 2002;47(6):610-3.

[3] Matsuo H, Tsunoda T, Ooyama K, Sakiyama M, Sogo T, Takada T, et al. Hyperuricemia in acute gastroenteritis is caused by decreased urate excretion via ABCG2. Scientific reports. 2016;6:31003.

[4] Takada Y, Matsuo H, Nakayama A, Sakiyama M, Hishida A, Okada R, et al. Common variant of PDZK1, adaptor protein gene of urate transporters, is not associated with gout. The Journal of rheumatology. 2014;41(11):2330-1.

Disclosure of Interest: None declared

DOI: 10.1136/annrheumdis-2017-eular.5673

\section{THU0049 29-KDA FIBRONECTIN FRAGMENT SUPPRESSED HMGB1-DEPENDENT AUTOPHAGY PATHWAY IN HUMAN ARTICULAR CHONDROCYTES}

M. Choi ${ }^{1}$, I.Y. Park ${ }^{1}$, K.M. Son ${ }^{2}$, H.S. Hwang ${ }^{1}$, H.A. Kim ${ }^{1} .{ }^{1}$ Hallym University Sacred Heart Hospital; ${ }^{2}$ Hallym University Dongtan Sacred Heart, Gyeonggi-do, Korea, Republic Of

Background: Fibronectin fragments found in synovial fluid induce the catabolic responses in cartilage. High mobility group protein Box 1 (HMGB1), a damageassociated molecular pattern (DAMP), resides in the nucleus, translocates into the cytosol in response to various stimuli, and is subsequently released into the extracellular space.

Objectives: In this study, we investigated the effect of $29-\mathrm{kDa}$ fibronectin fragment (29-kDa FN-f) on HMGB1-mediated autophagy pathway in primary human chondrocytes.

Methods: Human articular chondrocytes were enzymatically isolated from articular cartilage. The mRNA and protein levels of HMGB1 were measured by quantitative real-time PCR (qRT PCR) and Western blot analysis. The translocation of nuclear HMGB1 into cytoplasm was determined by western blot and immunofluorescence microscopy. Activation of mammalian target of rapamycin (mTOR), protein kinase $B$ (Akt) and 1A/1B-light chain 3 (LC3) was measured by western blot analysis. Interaction of HMGB1 and Beclin-1 were evaluated by immunoprecipitation (IP). Release of HMGB1 into extracellular medium was measured by ELISA.

Results: HMGB1 was significantly reduced in human osteoarthritis (OA) cartilage compared to normal cartilage. QRT PCR and Immunoblotting assay revealed that 29-kDa FN-f significantly reduced expression of HMGB1 at the mRNA and protein levels and also led to the translocation of the nuclear HMGB1 into the cytoplasm, together with decreased levels of Beclin-1 and phosphorylated Bcl-2. Using IP analysis, we demonstrated that in the presence of 29-kDa FN-f the association of HMGB1 and Beclin-1, which led to HMGB1-dependent autophagy pathway, was decreased, whereas the association of Bcl-2 and Beclin-1 was increased. In addition, prolonged treatment with $29-\mathrm{kDa} F N-f$ significantly increased the release of HMGB1 into the culture medium. A variety of evidence, including downregulated LC3-II, an autophagy marker, and increased phosphorylated 4EBP1 and p70S6K, substrates of mTOR, revealed that 29-kDa FN-f subsequently suppressed autophagy in primary chondrocytes by activating Akt/mTOR signaling pathway.

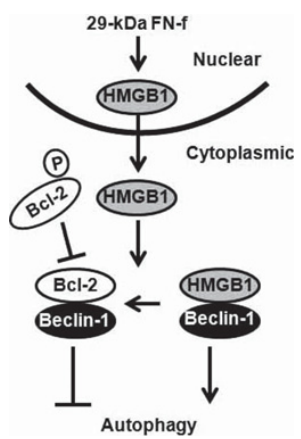

Conclusions: These results demonstrated that 29-kDa FN-f has a harmful impact on chondrocytes through suppressing HMGB1-dependent autophagy pathway and releasing HMGB1, DAMP, to extracellular space.

References:

[1] Kwak et al. Current Understanding of HMGB1-mediated Autophagy. J. Bacteriol. Virol. 43 (2013) 148-154.

[2] Heinola et al. High mobility group box-1 (HMGB-1) in osteoarthritic cartilage. Clinical. Exp. Rheumatol. 28 (2010) 511-518.

[3] Hwang et al. Fibronectin fragment-induced expression of matrix metalloproteinases is mediated by MyD88-dependent TLR-2 signaling pathway in human chondrocytes. Arthritis. Res. Therapy. 17 (2015) 320.

Acknowledgements: This study was supported by a grant $(\mathrm{HI} 14 \mathrm{C} 2248$ and HI15C2699) from the Korean Health Technology R\&D Project, Ministry of Health \& Welfare, Republic of Korea and the Basic Science Research Program through the National Research Foundation (NRF) of Korea funded by the Ministry of Education (2014R1A1A2059823)

Disclosure of Interest: None declared

DOI: 10.1136/annrheumdis-2017-eular.2812

\section{THU0050 MICRORNA-365 INHIBITS IL-1 $\beta$-INDUCED CATABOLIC FACTOR EXPRESSION BY TARGETING HIF- $2 \alpha$ IN HUMAN PRIMARY CHONDROCYTES}

M.H. Lee ${ }^{1}$, J.-I. Hong ${ }^{1}$, H.S. Hwang ${ }^{1}$, K.M. Son ${ }^{2}$, H.A. Kim ${ }^{1} .{ }^{1}$ Hallym University Sacred Heart Hospital, Kyunggi; ${ }^{2}$ Hallym University Dongtan Sacred Heart Hospital, Gyeonggi, Korea, Republic Of

Background: MicroRNAs, small noncoding RNA molecules, are involved in the pathogenesis of various diseases such as cancer and arthritis. Endothelial PAS 